\title{
Biomechanics of the transport barrier in the nuclear pore complex
}

\author{
George J. Stanley ${ }^{\mathrm{a}}$, Ariberto Fassati ${ }^{\mathrm{b}}$, and Bart W. Hoogenboom ${ }^{\mathrm{a}, \mathrm{c}, *}$ \\ ${ }^{a}$ London Centre for Nanotechnology, University College London, 17-19 Gordon Street, London \\ WC1H OAH, United Kingdom \\ ${ }^{\mathrm{b}}$ Division of Infection \& Immunity, UCL, Cruciform Building, 90 Gower Street, London WC1E 6BT, \\ United Kingdom \\ ${ }^{\mathrm{C}}$ Department of Physics and Astronomy, University College London, Gower Street, London WC1E \\ 6BT, United Kingdom
}

*Corresponding author: b.hoogenboom@ucl.ac.uk

\section{Abstract}

The nuclear pore complex (NPC) is the selective gateway through which all molecules must pass when entering or exiting the nucleus. It is a cog in the gene expression pathway, an entrance to the nucleus exploited by viruses, and a highly-tuned nanoscale filter. The NPC is a large proteinaceous assembly with a central lumen occluded by natively disordered proteins, known as FG-nucleoporins (or FG-nups). These FG-nups, along with a family of soluble proteins known as nuclear transport receptors (NTRs), form the selective transport barrier. Although much is known about the transport cycle and the necessity of NTRs for chaperoning cargo molecules through the NPC, the mechanism by which NTRs and NTR•cargo complexes translocate the selective transport barrier is not well understood. How can disordered FG-nups and soluble NTRs form a transport barrier that is selective, ATP-free, and fast? In this work, we review various mechanical approaches - both experimental and theoretical/computational - employed to better understand the morphology of the FG-nups, and their role in nucleocytoplasmic transport. Recent experiments on FG-nups tethered to planar surfaces, coupled with quantitative modelling work, suggest that FG-nup morphologies are the result of a finely balanced system with significant contributions from FG-nup cohesiveness and entropic repulsion, and from NTR•FG-nup binding avidity; whilst AFM experiments on intact NPCs suggest that the FG-nups are sufficiently cohesive to form condensates in the centre of the NPC lumen, which may transiently dissolve to facilitate the transport of larger cargoes.

\section{Keywords}

Nuclear pore complex; biomechanics; polymer physics; FG-nups; nucleocytoplasmic traffic.

\section{Table of Contents}

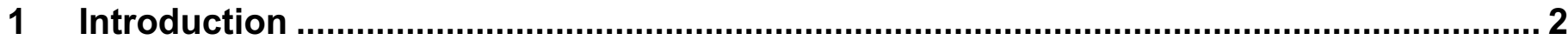

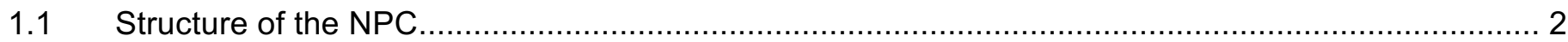

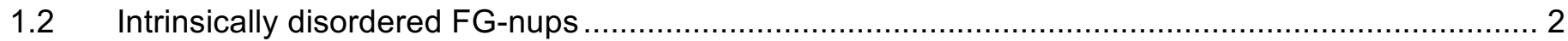

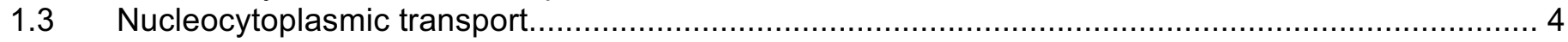

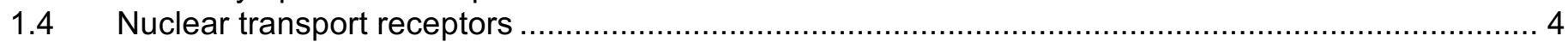

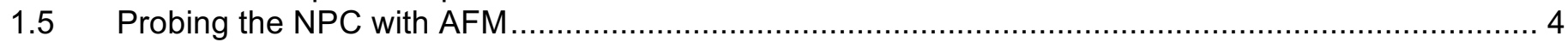

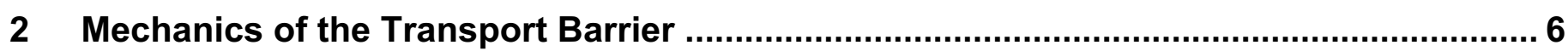

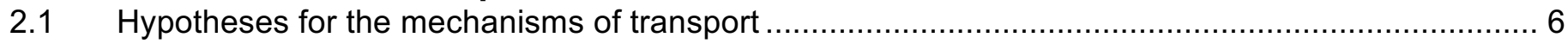

2.2 Polymer mechanics of FG-nups grafted in the planar geometry ..................................................

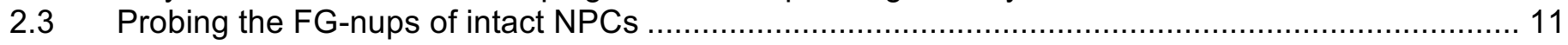




\section{Introduction}

\subsection{Structure of the NPC}

The nuclear pore complex (NPC) is a selective gateway for all macromolecules entering or exiting the nucleus. Small molecules can passively diffuse through the NPC (diameter $\lesssim 5 \mathrm{~nm}$ ), but larger molecules must bind to small chaperone proteins, called nuclear transport factors (NTRs), in order to translocate the NPC.

The NPC is a supramolecular, proteinaceous assembly, comprised of $\sim 30$ different nuclear pore proteins (hereby termed 'nucleoporins', or 'nups'), which assemble to form a pore across the nuclear envelope [1]. Each nucleoporin is present in many copies, to give a total of $\sim 1000$ nups, with a combined mass of $\sim 60-125 \mathrm{MDa}$ per pore [1-3]. The NPC has an eight-fold rotational symmetry around its central axis. At the cytoplasmic face of the NPC, eight filaments protrude into the cytoplasm; and at the nucleoplasmic face, a basket structure, also attached by protein filaments, extends into the nucleoplasm [4]. The NPC is modular: structural nucleoporins interact to form larger nup-subcomplexes, which in turn form the scaffold of the NPC. The structural scaffold of the NPC is made of three distinct rings: the cytoplasmic ring complex (CRC), the nucleoplasmic ring complex (NRC), and the inner ring complex (IRC) [3,5-10]. Both the CRC and the NRC are themselves made from two reticulated ring structures, comprised of 16 copies of the Y-shaped Nup107 subcomplex (in the case of the human NPC, Nup107 is formed from 10 nucleoporins [3]) [11]. These reticulated ring structures are then interlaced with other nups, and nup-subcomplexes, conferring structural heterogeneity between the CRC and the NRC. The IRC, although comprised of different nup-subcomplexes (Nup93 and Nup62 [12,13]), has a remarkably similar morphology to the CRC and NRC $[14,15]$. The Nup93 and Nup62 subcomplexes form Yshaped assemblies - akin to the Nup107 subcomplex - which intercalate to form a ring. The IRC then connects with the two outer rings (the CRC and NRC) via Nup155: a component of the Nup93 subcomplex $[14,15]$.

Of the $\sim 30$ nucleoporins that form the NPC, only about half are structural: forming the Y-shaped subcomplexes and other nup-subcomplexes intercalated into the outer and inner rings [14]. Anchored to the inner wall of the central channel of the NPC are many unstructured and intrinsically disordered nucleoporins. These disordered nups contain hydrophobic sequences rich in phenylalanine-glycine $(F G)$ repeats (hereby termed FG-nups: this term incorporates all hydrophobic sequences - such as FG, FxFG, and GLFG, amongst others - found in the disordered nups [16]). These are further discussed in section 1.2. From their anchoring sites at the inner wall of the NPC, the FG-nups emanate into the central channel and form the selective barrier to transport (see Figure 1a for a schematic of the NPC).

Although the nucleoporins between species can be very divergent (e.g., between the structurally integral Nup107 subcomplex in yeast and humans $[3,17])$, the overall architecture is conserved across all metazoans [18-21]. However, even with high-resolution data obtained for the scaffold structures of yeast, human, and Xenopus laevis NPCs [3,10,22], the disordered nature of the FGnups in the central channel means they have eluded the various structure-determination methods that depend on averaging techniques. In order to understand what morphology these FG-nups assume in the NPC, and how they can form a selective barrier to nucleocytoplasmic transport, other methods are required.

\subsection{Intrinsically disordered FG-nups}

The morphology of FG-nups in the central channel is of great importance for understanding the mechanism of selective transport through the NPC, and as such has been a topic of debate for 
many years [23-29]. The FG-nups occluding the central transport channel of the NPC are natively disordered [16,30,31]. As mentioned above, they contain repeating sequences of hydrophobic amino acids (such as FG, FxFG, FxFx, PSFG, and GLFG [16]), through which they can interact with one another, affording them a certain 'cohesiveness' [32] - with more cohesive FG-nups forming more compact morphologies, and less cohesive FG-nups forming more extended morphologies [26,32]. However, the conformation of FG-nups is not solely dependent upon the hydrophobic interactions between FG-domains. Repulsive, charged amino acids in the spacer regions of the FG-domains can counteract the cohesive interactions of the hydrophobic sequences [26]. A higher ratio of charged to hydrophobic amino acids in the FG-domain decreases cohesion and leads to protein extension; whilst a lower ratio affords increased cohesion and greater FG-nup compaction. It is probable that this heterogeneity in cohesion, compaction, and extension between the different FG-nups is used to nuance the NPC function along its axis of transport. For example, the glycosylated human Nup98, which is sufficiently cohesive to form hydrogels in vitro, and is found anchored to the inner ring of the NPC [12], may, in the confines of the cylindrical pore geometry, interact with FG-nups with diametrically opposed anchoring sites, to form a sizeexcluding meshwork - thus creating a selectivity barrier to transport. The human Nup153 however, located nearer the nuclear periphery [12], may, as well as comprising part of the transport barrier, act as a nucleation site for proteins required for the active transport of macromolecules [33]. However, the impact of FG-nup heterogeneity on the global morphology of FG-nups in the selective transport barrier deep inside the central channel is not well understood.
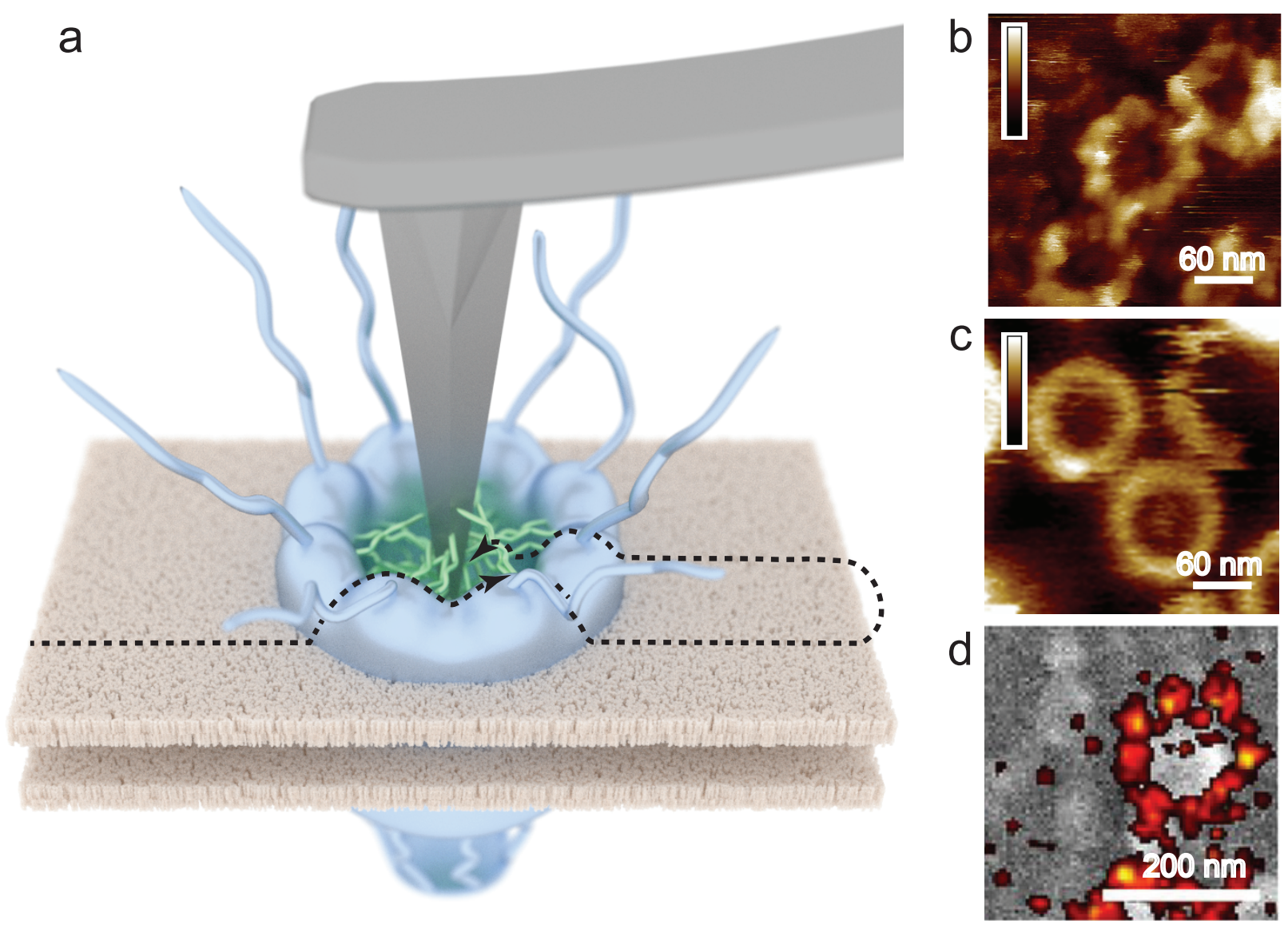

Figure 1. Imaging the nuclear pore complex. (a) Schematic of an AFM probe scanning line-byline over the cytoplasmic face of an NPC (not to scale). The dotted black line shows the path followed by the probe; at each pixel, the AFM tip indents $\sim 20 \mathrm{~nm}$ underneath the top surface of the NPC. High-aspect-ratio, supersharp silicon tips (radius $\sim 2 \mathrm{~nm}$ ) enable probing inside the central channel. Imaging is done in aqueous solution. (b) AFM image of the cytoplasmic side of the NPC in liquid with glutaraldehyde fixation, representing the eightfold symmetry of the NPC. (c) AFM image in liquid without glutaraldehyde fixation, demonstrating rotational symmetry at this spatial resolution. The NPC at the bottom exhibits the signature of a central plug in its height profile. The colour bar inset in both (b) and (c) is representative of $45 \mathrm{~nm}$ in height. (d) Correlative SEM- 
dSTORM with labelling of the luminal side of the transmembrane protein gp210. The image in (d) is adapted from Ref. [34], with permission (@ 2014, The Company of Biologists).

\subsection{Nucleocytoplasmic transport}

Although the NPC is the gateway through which all molecules must pass during nucleocytoplasmic transport, it is not the sole participant in the filtering process. The translocation of large molecules (diameter $\gtrsim 5 \mathrm{~nm}$, or mass $\gtrsim 40 \mathrm{kDa}$ ) is facilitated by complexation with other smaller proteins, called nuclear transport receptors (NTRs - also termed karyopherins, importins, exportins, and transportins). Nuclear transport receptors recognise and bind specific sequences of basic amino acids on the cargo proteins. These sequences are called nuclear localisation sequences (NLSs; for import) or nuclear export sequences (NESs; for export). Once bound, the affinity of the NTRs for the FG-nups in the NPC enables transport of the NTR•cargo complexes, via a mechanism that is not yet fully understood.

Furthermore, in this system a bias is required to ensure that once an NTR・cargo complex has completed a transport event, it does not (at least on average) re-enter the NPC and reverse its journey. This is accomplished by the 'Ran system'. At the end of an import event, a RanGTP molecule binds to the NTR of an NTR•cargo complex, inducing a conformational change and displacing the cargo molecule, thus releasing both the cargo molecule and the newly formed RanGTP•NTR complex into the nucleoplasm. This displacement reaction probably takes place whilst the NTR•cargo complex is interacting with Nup153 at the nuclear periphery of the NPC [33]. The RanGTP•NTR complex can then either return to the cytoplasm through the NPC, or bind to the nuclear export sequence of another cargo molecule, to facilitate its exit from the nucleus. Once in the cytoplasm, GTP hydrolysis of Ran by RanGAP and RanBP1 frees the cargo molecule and the NTR, leaving the hydrolysed RanGDP. This RanGDP:RanGTP gradient - with more RanGDP in the cytoplasm and more RanGTP in the nucleoplasm - is maintained by the nuclear RanGTPase RCC1, and is integral for maintaining directionality of transport across the NPC [35].

\subsection{Nuclear transport receptors}

NTRs belong to a family of proteins with approximately 20 members that all share similar properties with the prototype importin- $\beta$ (termed $\operatorname{Imp} \beta$ ): i.e., they can bind to RanGTP at the Nterminus, they are hydrophobic, and they exhibit an affinity for the FG-nups [36]. Imp $\beta$ is a $\sim 100$ kDa protein with $\sim 19$ HEAT-repeat sequences (i.e., antiparallel $\alpha$-helical domains) that interact with cargo molecules [37]. Imp $\beta$ has approximately 9 different FxFG binding sites: hydrophobic pockets that interact with the FG-nups [38]; the smaller transport receptor, NTF2 ( 29 kDa), has only two FxFG binding sites [39].

Although the role of NTRs in the import and export cycles is quite well understood, exactly how they facilitate the passage of cargoes through the FG-nups is not. NTRs, such as Imp $\beta$, have an affinity for the FG-nups. Experimental results suggest that transient, multivalent interactions occur between the FxFG binding sites and the FG-nups, with very fast binding and unbinding kinetics, perhaps explaining how NTRs can favour cargo molecules over inert cytosolic proteins, whilst maintaining a high rate of transport through the NPC (>100 transport events per second per NPC) $[31,40]$. At physiological concentrations ( $10 \mu \mathrm{M}[41])$, it is probable that $\operatorname{Imp} \beta$ is incorporated into the FG-nup meshwork inside the central conduit of the NPC [42]; and without Imp $\beta$, passive diffusion has been observed to increase [33]. This raises the question if - beyond their role in facilitating transport - NTRs are also essential for the barrier function of the NPC [43-45].

\subsection{Probing the NPC with atomic force microscopy}

The scaffold ring structures of the NPC have been quite well characterised by a variety of techniques $[3,7,10,15,22,46,47]$. However, the FG-nups, comprising the central transport barrier of 
the NPC, are largely disordered in nature and are therefore not very amenable to crystallographic or averaging methods. As a result, the morphology and workings of the FG-nups in the central transport barrier have not been elucidated, and there is scope for experimental methods that are complementary to conventional structural methods, such as X-ray crystallography and (cryo-) electron microscopy. One such technique is atomic force microscopy (AFM).

AFM is a surface technique that can build up an image by raster-scanning a sharp probe over a sample, line-by-line. As the AFM tip passes over the sample, it traces the sample contours, giving a topographical map of the material (see Figure 1), while monitoring the force between the tip and the sample via the deflection of the flexible cantilever upon which the tip is mounted. It can be done in aqueous solution, and does not require any chemical tagging or fixation (although fixation can help to immobilise samples). Over the years, this technique has been used to image the nuclear envelope of Xenopus laevis oocytes, both in air and in aqueous solution [48-61]. Further developments in AFM have enabled imaging at increasing spatial and temporal resolutions. Recently, this has led to the observation of time-dependent fluctuations of FG-nups, offering a glimpse of their dynamics at the cytoplasmic periphery of NPCs in aqueous solution [62]. At present, it is unclear as to what extent such observations can be extrapolated to the dynamics of FG-nups throughout the entire transport barrier. The barrier depth, in the case of NPCs in Xenopus laevis oocytes, is $\sim 50-60 \mathrm{~nm}$, compared to the sub-nanometre height scale at which dynamics were observed by AFM imaging. Therefore, for a greater understanding of the FG-nups' morphology and role in transport, information from below the surface is required.

Further to building an image, at every pixel, AFM can also mechanically indent below the top surface of the material, and the force response of the cantilever can be measured accordingly. This produces a force-distance curve (or force curve) at every pixel, which provides information on the nanomechanical properties of the material being indented. Therefore, using this technique, the elasticity and stiffness of the FG-nups can be measured, giving information on their morphology over more than $\sim 20 \mathrm{~nm}$ below the top surface of the transport barrier inside the NPC [28]. Are they interacting strongly as an ensemble to create a dense, hydrogel material? Are they interacting weakly and acting as predominantly entropic polymer brushes, thus occupying a larger but less dense volume? Or, are they somewhere in-between? Force curves can be collected, and thus nanomechanical properties recorded, at different locations with respect to the NPC scaffold structure. Analogous to procedures employed in electron microscopy, such data can be symmetrized by making use of the rotational symmetry of the NPC (limited, of course, by the spatial resolution within each experiment), and averaged for data collected over many NPCs. The resulting averages can be visualized by plotting stiffness cross-sections, which represent the force gradient experienced by the AFM tip as a function of vertical and radial position inside the NPC (see Figure 2). Such stiffness cross-sections are in good agreement with the NPC reconstructions as determined by cryo-electron microscopy [10], with the note that such reconstructions typically mask the very centre of the pore to facilitate the averaging process, and that they are less reliable in capturing disordered protein arrangements. 

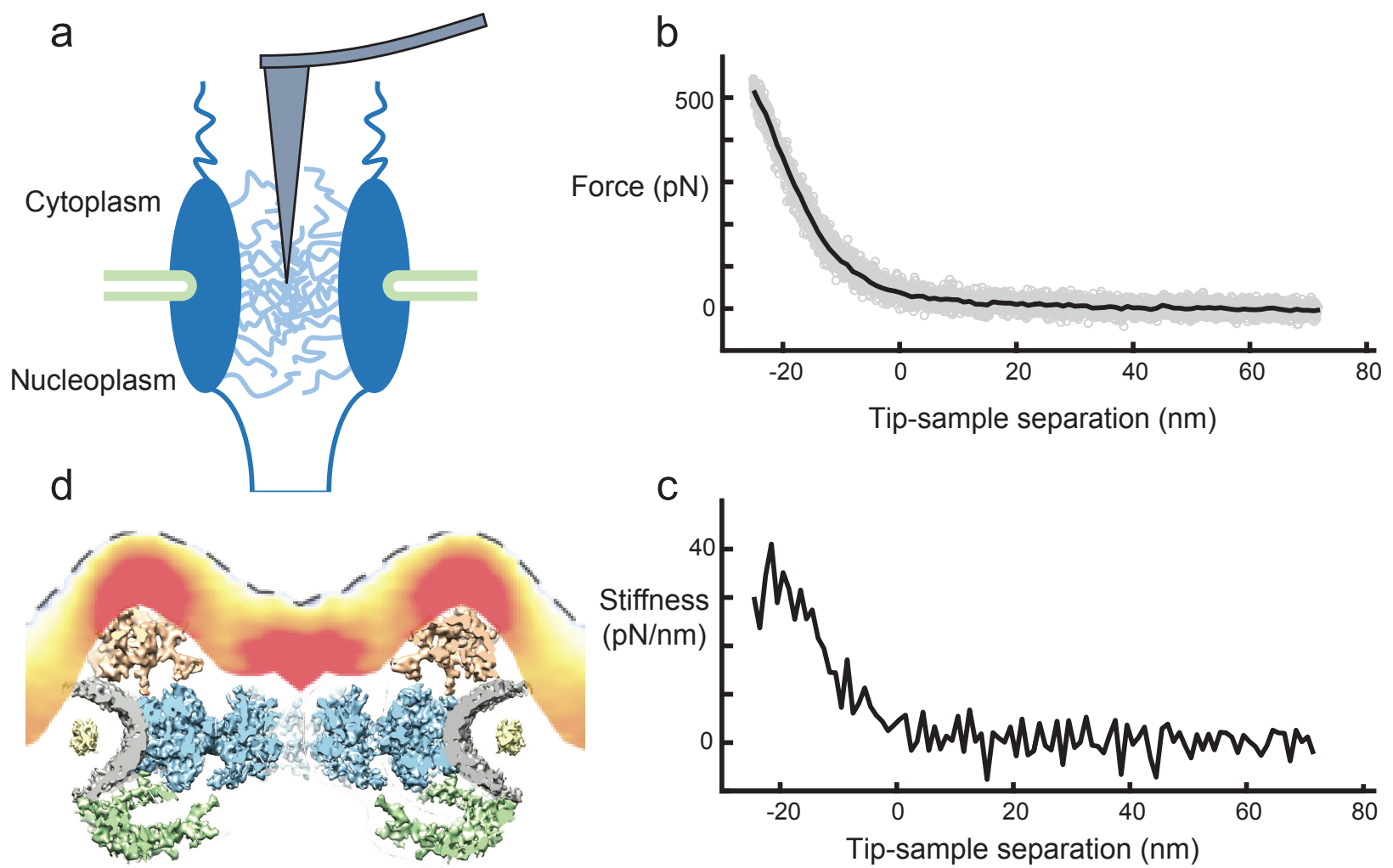

Figure 2. Nanomechanical mapping of the NPC. (a) Schematic showing an AFM tip probing inside the central transport channel of the NPC (not to scale). (b) The force curve (black) obtained after averaging (here: $n=9$ ) force curves recorded in the central channel of an NPC. Grey circles indicate the non-averaged data points. (c) Stiffness curve as obtained by a numerical derivative of the force curve in b. (d) The stiffness cross-section of the cytoplasmic face of the NPC (from Ref. [28], with permission, (C) 2014, Nature Publishing Group), overlaid on an EM reconstruction (adapted from Ref. [10], licensed under CC-BY 4.0). Force curves are collected and averaged based on their radial position with respect to the central axis of rotation of the NPC. This symmetrises the NPC, and renders a profile representation. Red represents a greater stiffness response from the material, and yellow a smaller stiffness response.

\section{Mechanics of the Transport Barrier}

\subsection{Hypotheses for the mechanisms of transport}

The precise role played by FG-nups and NTRs in the transport of cargoes is not easy to resolve. NTRs are known to have an affinity for FG-nups, NLSs, and NESs, and to promote the passage of cargoes; but the precise morphology of FG-nups is not known. It is also apparent that the rate of nucleocytoplasmic transport is very fast: it has been estimated that a mass of nearly $100 \mathrm{MDa}$ translocates a single NPC every second [63]. However, one can imagine that if the binding interaction between NTRs and FG-nups is too strong, the rate of transport will be slow; but that if it is too weak, cargo molecules will not be sufficiently favoured over inert proteins. How is it that transport through the NPC can be both fast and selective?

This debate has manifested itself through various streams of thought, which may be positioned on a scale that ranges between two extremes: one, in which the FG-nups are assumed to be cohesive to the extent of forming a hydrogel, or a size-selective meshwork; and the other, in which the FG-nups are not cohesive, but instead form a predominantly entropic barrier to transport, due to the reduction in configurational freedom (and thus in entropy) experienced by FG-nups upon interaction with cargo molecules. The selective phase model, for example, assumes that FG-nups are cohesive enough to form a hydrogel or a cross-linked meshwork, with a mesh size that allows 
passive diffusion of small molecules ( $\$ 5 \mathrm{~nm}$ in diameter) but excludes larger, inert molecules. NTRs can then interact with the FG-nups to melt through the meshwork, shuttling bound cargo molecules [24,63-68]. On the other hand, the virtual gate model assumes a lesser degree of cohesion between the FG-nups. In this scenario, the FG-nups act as polymer brushes that exert entropic forces against compression, which provides a barrier to inert molecules. Stochastic binding events between NTRs and FG-nups then enable the passage of NTR•cargo complexes through the NPC $[29,31,69-71]$. Similarly, the reduction-of-dimensionality mechanism assumes that FG-nups are not sufficiently cohesive to form a meshwork across the entire diameter of the pore. Instead, a wall of hydrophobic, dynamic FG-nups covers the inner walls of the NPC, leaving a small central channel un-occluded in the centre of the pore to allow passive diffusion.

NTR•cargo complexes then bind to the hydrophobic wall of FG-nups, and conduct a 2D, or nearly 1D random walk to pass through the NPC $[25,27,72]$. The forest model suggests that more charged, less cohesive domains cause FG-nups to extend from the NPC scaffold towards the central axis, acting as entropic polymer brushes. This extension positions the more cohesive domains of FG-nups towards the NPC centre, where they can form a hydrogel. This leaves various possible routes of transport for NTR•cargo complexes $[10,26]$. The list of proposed mechanisms goes on $[44,73,74]$.

Although some studies still suggest that the selection of passage through the NPC is dominated by entropic effects [29,31], other recent work indicates that the morphology of the FG-nups exhibits both FG-nup cohesion and entropic repulsion [42,75], the two of which may be finely tuned to facilitate the selective transport of NTRs and NTR•cargo complexes.

\subsection{Polymer mechanics of FG-nups grafted in the planar geometry}

Due to the difficulty of probing FG-nups in the cylindrical geometry (whether in intact NPCs or biomimetic nanopores), several experiments have been performed on FG-nups tethered to a planar surface, thereby making them more amenable to surface techniques. By performing these experiments, several questions can be addressed: Do the FG-nups extend away from the substrate and into solution (i.e., do they act as predominantly entropic and steric polymer brushes), or do they collapse towards the substrate (i.e., forming a strongly cohesive and therefore condensed polymer brush with properties approaching those of a hydrogel)? Does the FG-nup film swell or collapse upon addition of NTRs? And, is the observed behaviour dependent upon parameters such as grafting density (i.e., the concentration of FG-nups inside the NPC), FG-nup cohesiveness, NTR•FG-nup binding avidity, and NTR size and concentration?

Initial experiments in this area gave conflicting results. A study by Lim et al. (2007), using the FxFG-rich domain of human Nup153 - found at the nuclear periphery of the NPC [12] - reported that the FG-nups form an entropic polymer brush. Upon addition of mammalian Imp $\beta$, at concentrations several orders of magnitude below that of physiological concentrations [73], the film was observed to collapse dramatically towards the substrate. However, a similar study by Eisele et al. (2010) did not show any sign of a collapse [76]. In this instance, the FxFG-domain of the yeast FG-nup, Nsp1p - found both in the central channel and at the nuclear periphery of the NPC $[77,78]$ - was tethered to a supported lipid bilayer. Upon addition of low concentrations of yeast Imp $\beta(\sim 1 \mu \mathrm{M})$, hardly any change was observed in film thickness, but upon addition of physiological concentrations of $\operatorname{Imp} \beta(\sim 10 \mu \mathrm{M}$ [41]), the FG-domain was observed to swell by a few nanometres, from its original film thickness of tens of nanometres.

The contradiction between these studies highlights the importance of parameters such as FG-nup grafting density, FG-nup cohesiveness, NTR•FG-nup binding avidity, and NTR concentration. The extension of any given FG-nup away from a substrate is not an intrinsic property of the protein, but is dependent upon all these parameters [75]. The human Nup153 is likely less cohesive than the yeast Nsp1. Furthermore, in the experiments mentioned above, the grafting density of Nup153 was far lower than that of Nsp1p; the binding avidity of Nup153 to mammalian Imp $\beta$ could be as 
much as an order of magnitude stronger than that of Nsp1 to yeast Imp $\beta[79,80]$; and the concentration of mammalian Imp $\beta$ added to Nup153 was far less than the concentration of yeast $\operatorname{Imp} \beta$ added to Nsp1p. Further complications may arise from differences in the underlying substrate and its passivation.

Indeed, without considering the contribution made by the NTRs, but instead probing the FGdomain films as a function of FG-nup cohesiveness and grafting density, the behaviour of the proteins can begin to be elucidated [32]. In a study by Eisele et al. (2013), three different FGdomain films, each with varying degrees of cohesiveness, were tethered to a supported lipid bilayer: the very cohesive O-GIcNAc modified Nup98 from Xenopus tropicalis, 'Nup98-glyco'; the slightly less cohesive Nsp1-WT from Saccharomyces cerevisiae; and the relatively non-cohesive Nsp1-FILV $\rightarrow$ S mutant [32]. It was observed that the binding kinetics of the FG-domains to the supported lipid bilayer substrate depended on surface occupancy, but more importantly on FGdomain cohesiveness. The very cohesive Nup98-glyco exhibited fast binding kinetics up to a surface coverage $(\Gamma)$ of $10 \mathrm{pmol} / \mathrm{cm}^{2}$, the less cohesive Nsp1-WT exhibited an appreciable decrease in binding rate after $\Gamma=6 \mathrm{pmol} / \mathrm{cm}^{2}$, and for the non-cohesive Nsp1-FILV $\rightarrow S$ mutant, the surface coverage plateaued at $\sim 5 \mathrm{pmol} / \mathrm{cm}^{2}$ [32]. These results can be understood in terms of FGdomain interaction strength. For the more cohesive FG-nups, the entropic penalty incurred from a higher grafting density is compensated by the stronger FG-domain interactions; but for the less cohesive FG-nups, the entropic penalty of a high grafting density is too high, and cannot be compensated by the weaker FG-domain interactions. Furthermore, the self-assembled FG-domain morphology is dependent upon the grafting density. AFM imaging revealed homogeneous hydrogels for the cohesive Nup98-glyco at grafting densities of $\sim 9 \mathrm{pmol} / \mathrm{cm}^{2}$, but at $4 \mathrm{pmol} / \mathrm{cm}^{2}$ a heterogeneous surface was observed, suggesting disruption to the FG-domain morphology, and regions of partial collapse [32].

However, it is important not to interpret these results through the binary lens of either a hydrogel or a polymer brush. As Eisele et al. (2013) comment in their work, by defining the term hydrogel as a 'nonfluid polymer network that is expanded throughout its whole volume by aqueous solvent', it can be imagined that there is a sliding scale from entropic-brush to gel-like morphologies of FGnups [32]. The FG-nups can exhibit the behaviour of cohesive polymers, forming strong, nontransient cross-links, and a stable nonfluid hydrogel; they can be less cohesive polymers, forming weaker, transient cross-links, creating a less-dense fluid hydrogel; or, if they are non-cohesive or even repulsive polymers, they can sample lateral as well as extended states, forming a polymer brush that is dominated by entropic and steric interactions, but perhaps still experiencing interpenetration, therefore forming a voluminous, larger meshwork. Indeed, FRAP results gave further evidence to this idea [32]. By watching the lateral diffusion of the three different FG-domain films tethered to a fluid SLB, it was observed that the very cohesive Nup98-glyco showed very little lateral diffusion; a slow but significant rate of diffusion was observed for the less cohesive Nsp1WT in the same time-frame; and very quick diffusion was observed for the non-cohesive Nsp1FILV $\rightarrow$ S. The varying levels of cohesiveness were forming morphologies with different degrees of interpenetration: ranging from stable, nonfluid hydrogels (Nup98-glyco), to mobile, fluid polymer brushes (Nsp1-FILV $\rightarrow$ S), with Nsp1-WT somewhere in the middle.

More recently, models based upon polymer physics have begun to more quantitatively elucidate the behaviour of FG-domain films on planar surfaces, converging on the idea that their morphology is probably the result of a finely balanced system exhibiting significant contributions from both FG-nup•FG-nup cohesion and entropic repulsion [42,75]. A study by Zahn et al. (2016), monitoring the binding kinetics of the Homo sapiens NTF2 with three different FG-domains - Nsp1 from Sacchromyces cerevisiae, Nup98-glyco from Xenopus tropicalis, and a synthetic polypeptide with regular FSFG repeat domains; as well as Sacchromyces cerevisiae Importin- $\beta$ with Nsp1 has shown that remarkably similar binding kinetics are observed for different NTRs with different FG-domains. For both NTRs, and for all FG-domains, a similar shape to the binding isotherms was observed: always exhibiting fast initial binding kinetics, followed by negative cooperativity in the physiologically relevant concentration range (phenomena also experimentally observed in other 
studies [44,45,74]). Similarly, another model developed by Vovk et al. (2016), also based upon polymer physics, has arrived at a qualitatively similar result, namely, that the cohesiveness of the FG-nups is tuned to be somewhere in-between the ideal non-cohesive polymer brush morphology, and the cohesive, nonfluid, hydrogel [75]. Using the experimental data from Kapinos et al. (2014) and Wagner et al. (2015) [44,45], for Nup62, Nup98, Nup153, and Nsp1 tethered to a planar surface, the morphology of all the FG-nups was found to be in-between the two extreme regimes (polymer brush and hydrogel) [75]. This raises the question: Is heterogeneity in FG-nups and in NTRs important to the transport barrier of the NPC, or do more generic principles govern the rate and selectivity of transport through the NPC?

Without considering the heterogeneity between the FG-nups, and only considering two parameters - the FG-nup cohesiveness and NTR•FG-nup binding avidity - the model developed by Zahn et al. (2016), accurately reproduced the experimental results [42]. It is therefore possible that general biophysical principles determine transport through the NPC. Whilst it is true that heterogeneity in the FG-nups may nuance their function along the axis of transport, it may only be of minor importance for the FG-nups forming the selective transport barrier deep inside the central channel. For these FG-nups, it is conceivable that the overall balance between cohesive and repulsive FGdomains, and the strength of NTR•FG-nup binding avidities, dictates their function. If one imagines both the cohesive properties of the FG-nups and the NTR•FG-nup binding avidities as averaged-out properties, the mechanism of selective and fast transport can be reasoned. The FGnup cohesiveness is tuned to be great enough to create a size-excluding meshwork (thus excluding large, inert proteins), but weak enough so that it can be interpenetrated by NTRs; whilst the NTR•FG-nup binding avidity is tuned to be strong enough to displace FG-nup•FG-nup interactions, but weak enough to have fast unbinding kinetics, and maintain fast rates of transport through the NPC. The selectivity of the NPC can also be explained. As shown by the modelling work [42], a small decrease in the NTR•FG-nup binding avidity engenders a drastic drop in binding kinetics, thus excluding inert proteins which have a weak affinity for the FG-nups. 

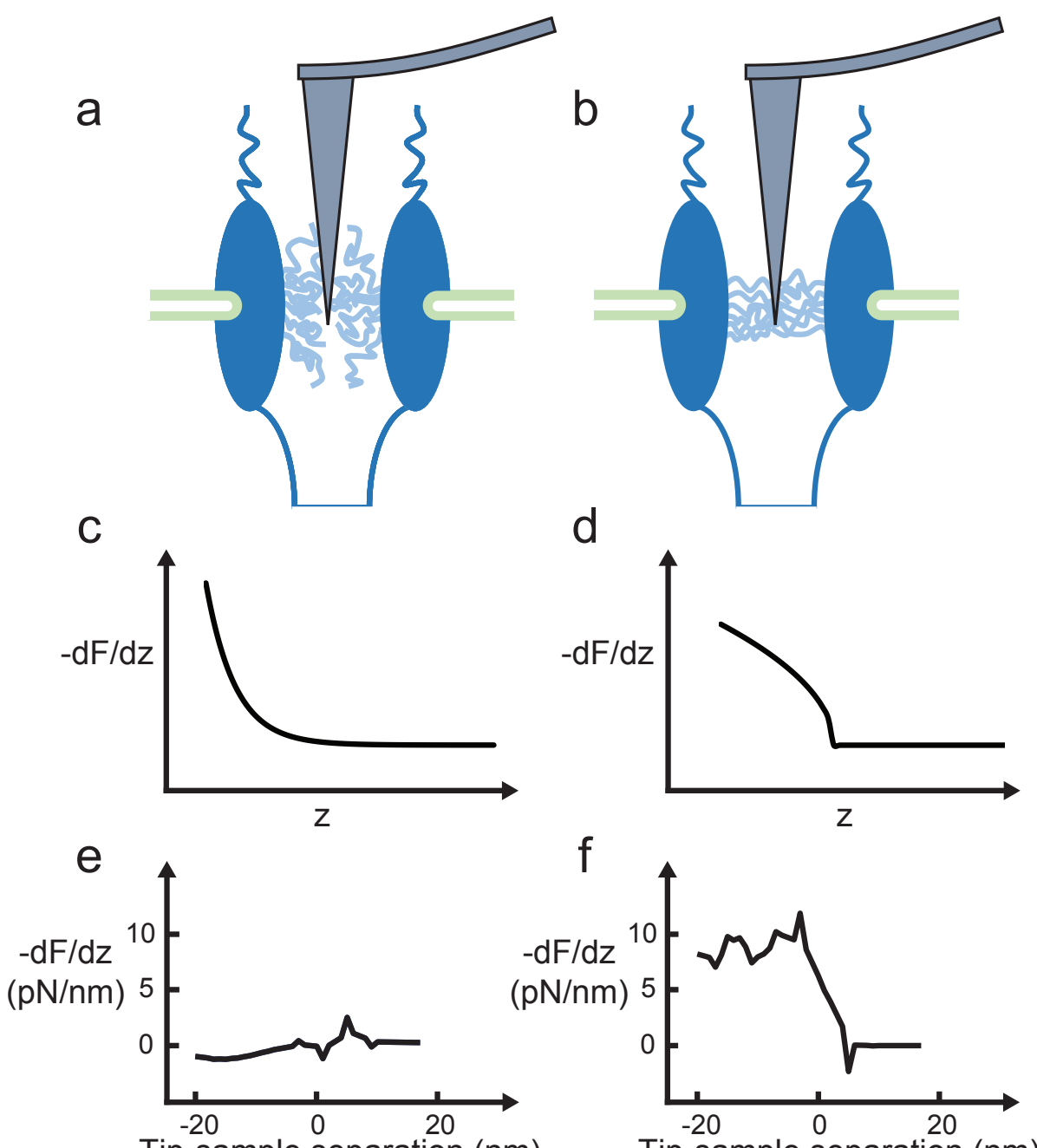

Tip-sample separation $(\mathrm{nm})$

Tip-sample separation (nm)

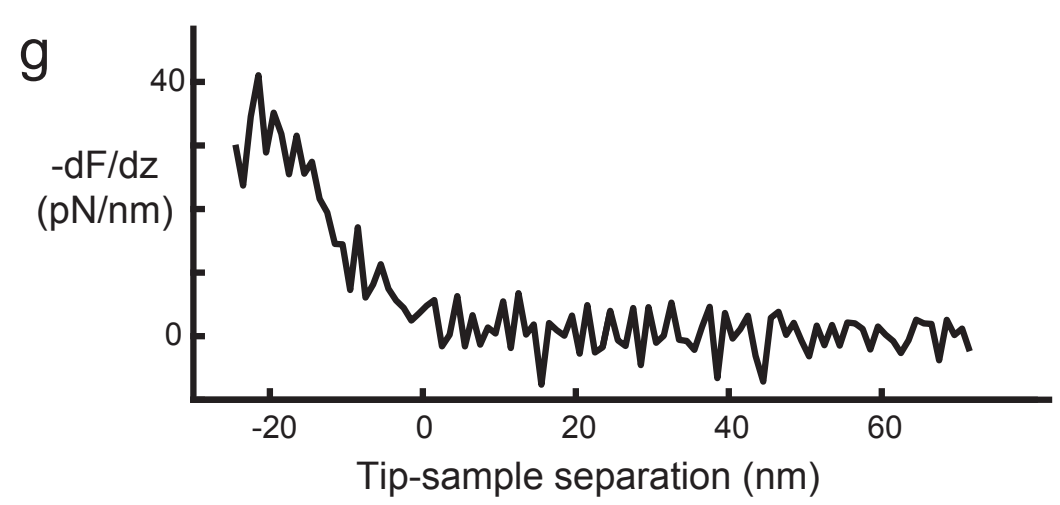

Figure 3. Mechanical characteristics of polymer brush and hydrogel models. (a) Schematic of the NPC showing the central nups in the ideal polymer brush conformation. (b) Schematic of the NPC with the nups in the dense hydrogel conformation. (c) Predicted (qualitative) shape of a stiffness curve for an AFM tip interacting with polymer brush nups. (d) Predicted (qualitative) shape of a stiffness curve for FG-nups forming a hydrogel. (e) Stiffness curve obtained from a DFT calculation for an AFM tip indenting into FG-nups that are just not sufficiently cohesive to condense in the central NPC channel, resulting in entropic-brush characteristics. (f) Stiffness curve obtained from a DFT calculation for an AFM tip indenting into FG-nups that are just sufficiently cohesive to condense in the central NPC channel, resulting in mechanical characteristics indicative of a hydrogel. (g) Experimentally obtained stiffness curve obtained from the central channel of the NPC (cytoplasmic side). Overall, mechanical data on intact NPCs [28] are in closer agreement with a hydrogel model, which does not exclude, however, an entropic contribution. DFT curves are adapted from Ref. [28], with permission (C) 2014, Nature Publishing Group). 


\subsection{Probing the FG-nups of intact NPCs}

As outlined above, it is difficult to directly probe the FG-nups of intact NPCs. The diameter of the NPC transport channel is $\sim 40 \mathrm{~nm}$, with many of the FG-nups located deep inside the lumen; in addition, the nucleoplasmic face of the NPC is occluded by the nuclear basket, meaning it cannot be probed by surface techniques. However, by using AFM with a supersharp, high-aspect-ratio silicon probe, with a tip radius of $\sim 2 \mathrm{~nm}$, the FG-nups can be accessed via the cytoplasmic face of the NPC. Furthermore, by indenting below the top surface of the FG-nups (over a depth >20 nm) and recording the force response of the cantilever (as explained in section 1.5), conclusions can be drawn about the type of material being probed [28]. For example, is the material acting as a hydrogel, or as a polymer brush? Or is it somewhere in-between?

Figure $3 a$ and $3 b$ show schematics of the FG-nups in the idealised polymer brush and dense hydrogel morphologies, respectively. Also shown are the stiffness curves as qualitatively predicted to be rendered if the FG-nups assumed either of these extreme morphologies (Figure 3c and 3d); the (quantitative) stiffness curves calculated from polymer physics (classical DFT) for conformations at the crossover between the two extremes (Figure $3 e$ and 3f) [81,82]; and the stiffness curve experimentally obtained from the central transport channel of the NPC in aqueous solution (Figure $3 \mathrm{~g}$ ). The experimental curve demonstrates a far greater stiffness than is predicted for the predominantly entropic polymer brush scenario, and shows a general shape more similar to that calculated for the hydrogel morphology. These results suggest that the cohesion of the FGnups is strong enough to form some sort of clump, or condensate of FG-nups in the centre of the NPC transport channel, albeit only marginally so (which would make sense, as the condensate would need to be dissolved to facilitate transport of larger cargoes). Modelling work suggests that, in the cylindrical geometry, this centrally condensed morphology of polymers becomes increasingly more likely as the cohesiveness of the FG-nups increases $[81,83]$.

However, in native NPCs, it must be considered that NTRs may form an intrinsic component of the transport barrier (as discussed in section 1.4). Indeed, experiments of NTRs binding to FG-nup planar films (at relevant polymer grafting densities and physiological concentrations of NTRs) affords an increase in FG-domain mass, sometimes by as much as 40\% [42]: suggesting that the FG-nups are both interpenetrated by, and shrouded in NTRs, and that the transport barrier in vivo is likely to contain large volume fractions of both FG-nups and NTRs at all times (schematics of possible NTR/FG-nup arrangements in the NPC are shown in Figure 4a and 4b). In order to probe the contribution of NTRs to the transport barrier, NPCs were incubated with the Ran system and ATP (to activate the transport cycle and flush out NTRs and cargos), and benzonase (to digest ribonucleoproteins: a common cargo molecule), and their stiffness cross-sections were measured (as outlined in section 1.5) [28]. In spite of a significant reduction of cargo molecules in the nuclear envelope (as measured by fluorescence microscopy and Western blot analysis), the measured stiffness profile was similar to that of a paired control (Figure 4c). However, a stiffness contribution from NTRs could not be fully excluded in these experiments. The stiffness response observed from the central channel, even after activation of the transport cycle, implies that the FG-nup condensate still forms in the central transport channel, i.e., the cohesiveness of the FG-nups is great enough to form a central plug, albeit that this may be facilitated by the presence of NTRs remaining in the barrier. Upon deliberate incubation of the NPCs with exogenous $\operatorname{Imp} \beta$, a swelling of the transport channel was observed, along with homogenisation of the stiffness cross-section along the radial profile of the NPC (Figure 4d). This swelling behaviour, also observed on planar FG-domain films $[42,44,45,76]$, suggests an accumulation of Imp $\beta$ both in and around the FGnups. Possible NTR/FG-nup arrangements for such accumulation have been proposed by physical modelling, again dependent upon FG-nup cohesiveness and NTR•FG-nup binding avidity, and remain to be tested [82].

Taken as a whole, experiments on FG-domain planar films, and AFM experiments on intact NPCs, coupled with modelling work, suggest that the FG-nups within the NPC assume a morphology 
resulting from a finely tuned system balanced by contributions from FG-nup cohesion and entropic repulsion, and NTR•FG-nup binding avidity. The affinity of the NTR•cargo complexes for the FGnups, and their fast binding and unbinding kinetics, then allows for the fast and selective transport of cargoes through the NPC. However, this does not explain how large cargoes, i.e. those approaching the size of the pore diameter (such as virus capsids [84]), translocate the NPC. Figure $5 a, 5 b$ and $5 c$ show the results from Monte Carlo simulations of polymers in the pore geometry, with the same cohesiveness, but different starting conformations $[81,85]$, to outline behaviours that may be adopted by FG-nups in the NPC. The polymers can find two different (meta)stable morphologies: the central polymer condensate (Figure 5a) or the collapsed wall state (Figure $5 \mathrm{~b}$ and $5 \mathrm{c}$ ). This raises the attractive idea that the cohesive properties of the FG-nups are tuned to be near the phase boundary between these two states. This would enable the FG-nups to natively maintain a morphology akin to the central phase (Figure 5a), thereby occluding the central transport channel to maintain the selective barrier; but to then be able to drastically rearrange and assume a morphology similar to the wall phase (Figure $5 b$ and $5 c$ ), with only a small activation energy barrier, thus facilitating the transport of large cargoes. Indeed, best agreement between experimental data and model predictions is found for an FG-nup cohesiveness that is only just sufficient to form a central FG-nup condensate in the NPC channel [28].

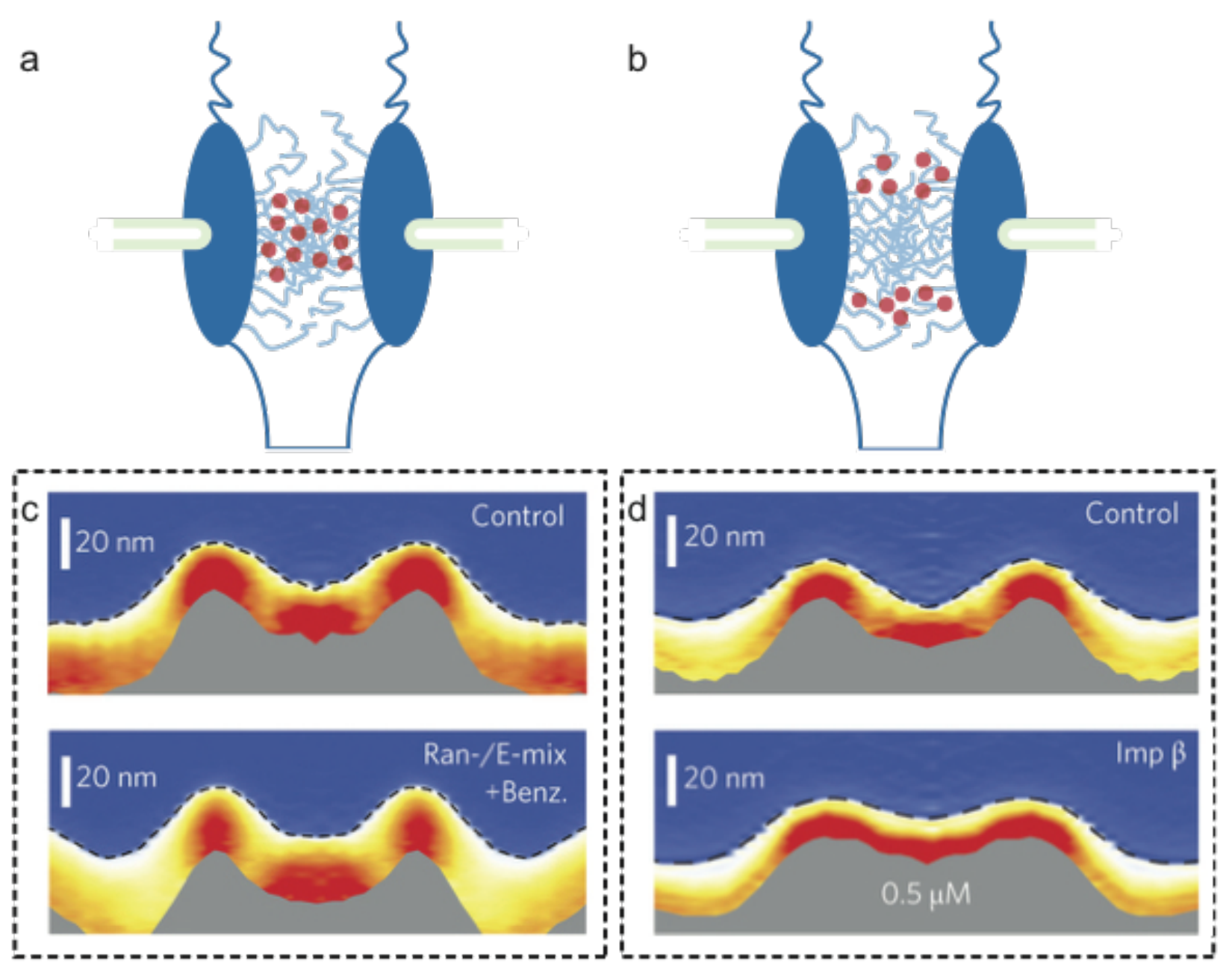

Figure 4. Measuring mechanical properties inside the NPC. (a) Schematic of the NPC showing a possible arrangement for the nuclear transport receptors (red circles) interacting with the FGnups [82]. The NTRs form a network within the polymer meshwork. (b) Schematic of the NPC showing another possible conformation for the NTRs interacting with the nups. Here, the increased FG-nup•FG-nup interaction strength (as compared to the NTR•FG-nup interaction strength) results in the NTRs being on average localized above and below the nups. (c) Averaged stiffness cross-sections of NPCs in liquid following washes without (control; top) and with Ran-/Emix and benzonase (bottom). The Ran-/E-mix and benzonase flushes out nuclear transport receptors and digests ribonucleoproteins (cargoes) stuck in transit. (d) Averaged stiffness crosssections of NPCs in liquid following washes without additional NTRs (control; top), and with added 
Importin- $\beta$ ( $0.5 \mu \mathrm{M}$; bottom). Stiffness cross-sections adapted from Ref. [28], with permission (C) 2014, Nature Publishing Group).

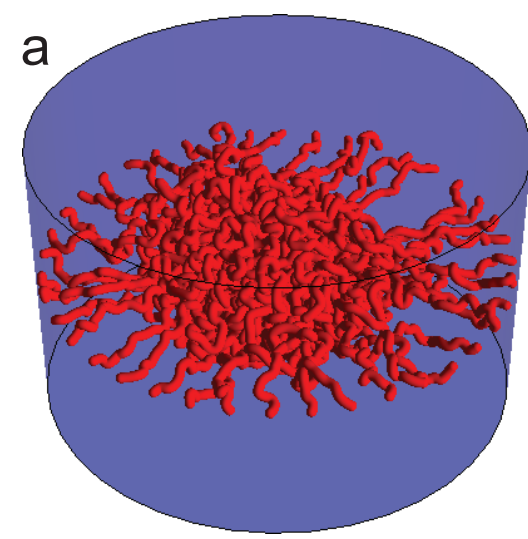

Central Phase

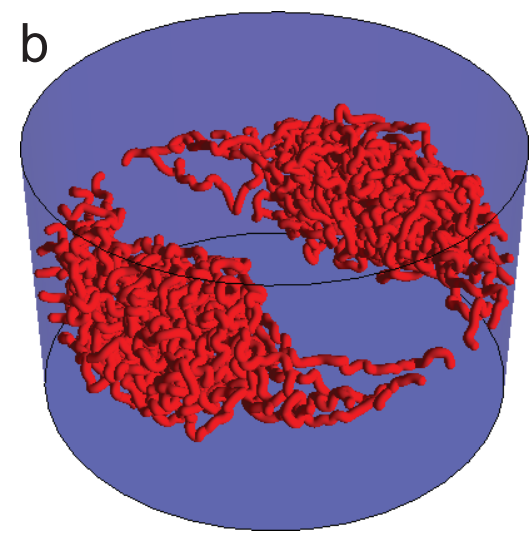

Wall Phase

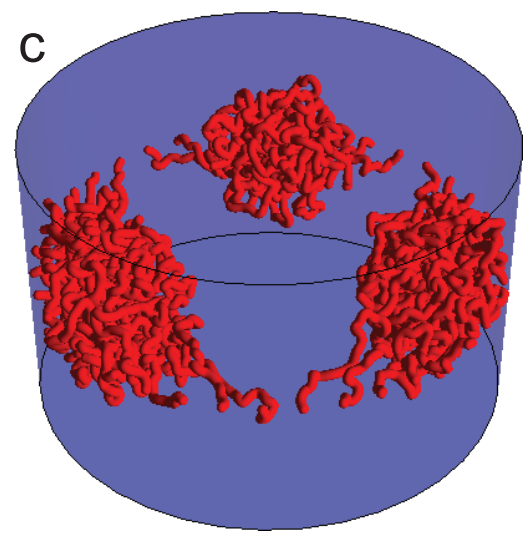

Wall Phase

Figure 5. Monte Carlo simulations of polymers in a cylinder. With the same parameters but different starting conditions, Monte Carlo simulations of polymers anchored inside a cylinder show two distinctly different conformations: the central phase (a) and the wall phase (b \& c). 40 polymers of length $100 \mathrm{~nm}$ and segment length $1 \mathrm{~nm}$ are simulated in a cylinder of radius $25 \mathrm{~nm}$. Figure adapted from Ref. [85], licensed under CC-BY 3.0.

\section{Concluding Remarks}

For many years, the debate surrounding the role played by FG-nups and NTRs in the formation of the selective transport barrier has focussed on the differences between (predominantly) cohesive versus entropic behaviour of FG-nups in reconstituted model systems and in the NPC. While recent studies to some extent continue to primarily emphasise entropic $[29,31]$ or cohesive $[28,32,42,86]$ aspects of the transport barrier, quantitative analyses of experimental data now indicate that both cohesion and entropic repulsion have significant roles to play $[42,75]$ and are certainly not mutually exclusive. Quite generally, entropic aspects are undeniably essential to determine the morphology of unstructured, flexible proteins such as FG-nups; and a certain degree of FG-nup cohesion may even be required for the biogenesis of the NPC: binding of noncohesive FG-nups to a supported lipid bilayer exhibit very slow kinetics [32], and result in grafting densities that would not account for the concentration of FG-repeat domains found in mammalian NPCs [87].

Focussing on the polymer mechanics of FG-nup assemblies, one may hypothesise that NPC transport selectivity results from the rather finely tuned balance between cohesive and entropic interactions [42,75]. Indeed, mechanical measurements of FG-nups in NPCs [28] suggest a cohesiveness that is just about sufficient to enable them - possibly facilitated by uptake of NTRs to span across and thus seal the transport channel, thereby creating a selective transport barrier. Fast rates of transport would thus be facilitated by the vicinity to transitions between different FGnup morphologies [81,85], as well as by the fast, multivalent binding and unbinding kinetics between the FG-nups and the NTRs [40]; whilst the affinity of the NTRs to the FG-nups engenders the selectivity, favouring NTR•cargo complexes over inert macromolecules. Over the following years, biophysical studies are likely to further validate such hypotheses and lead to a more quantitative understanding of transport selectivity of nuclear import and export.

\section{Acknowledgements}


The authors acknowledge Adrian Hodel for assistance in preparing Figure 1. This work was supported by the UK Biotechnology and Biological Sciences Research Council (BB/J014567/1).

\section{References}

[1] J.M. Cronshaw, A.N. Krutchinsky, W. Zhang, B.T. Chait, M.J. Matunis, Proteomic analysis of the mammalian nuclear pore complex, J. Cell Biol. 158 (2002) 915-27. doi:10.1083/jcb.200206106.

[2] R. Reichelt, A. Holzenburg, E.L. Buhle Jr, M. Jarnik, A. Engel, U. Aebi, Correlation between Structure and Mass Distribution of the Nuclear Pore Complex and of Distinct Pore Complex Components, J. Cell Biol. 110 (1990) 883-894.

[3] K.H. Bui, A. Von Appen, A.L. Diguilio, A. Ori, L. Sparks, M.T. Mackmull, T. Bock, W. Hagen, A. AndrésPons, J.S. Glavy, M. Beck, Integrated structural analysis of the human nuclear pore complex scaffold, Cell. 155 (2013) 1233-1243. doi:10.1016/j.cell.2013.10.055.

[4] M. Jarnik, U. Aebi, Toward a more complete 3-D structure of the nuclear pore complex, J. Struct. Biol. 107 (1991) 291-308. doi:10.1016/1047-8477(91)90054-Z.

[5] C.W. Akey, M. Radermacher, Architecture of the Xenopus nuclear pore complex revealed by threedimensional cryo-electron microscopy, J. Cell Biol. 122 (1993) 1-19. doi:10.1083/jcb.122.1.1.

[6] M. Beck, F. Förster, M. Ecke, J.M. Plitzko, F. Melchior, G. Gerisch, W. Baumeister, O. Medalia, Nuclear Pore Complex Structure and Dynamics Revealed by Cryoelectron Tomography, Science. 306 (2004) 1387-1390.

[7] M. Beck, V. Lučić, F. Förster, W. Baumeister, O. Medalia, Snapshots of nuclear pore complexes in action captured by cryo-electron tomography, Nature. 449 (2007) 611-615. doi:10.1038/nature06170.

[8] D. Frenkiel-Krispin, B. Maco, U. Aebi, O. Medalia, Structural Analysis of a Metazoan Nuclear Pore Complex Reveals a Fused Concentric Ring Architecture, J. Mol. Biol. 395 (2010) 578-586. doi:10.1016/j.jmb.2009.11.010.

[9] T. Maimon, N. Elad, I. Dahan, O. Medalia, Article The Human Nuclear Pore Complex as Revealed by Cryo-Electron Tomography, Structure. 20 (2012) 998-1006. doi:10.1016/j.str.2012.03.025.

[10] M. Eibauer, M. Pellanda, Y. Turgay, A. Dubrovsky, A. Wild, O. Medalia, Structure and gating of the nuclear pore complex, Nat. Commun. 6 (2015) 7532. doi:10.1038/ncomms8532.

[11] E. Hurt, M. Beck, Towards understanding nuclear pore complex architecture and dynamics in the age of integrative structural analysis, Curr. Opin. Cell Biol. 34 (2015) 31-38. doi:10.1016/j.ceb.2015.04.009.

[12] S. Krull, J. Thyberg, B. Bjo, H. Rackwitz, V.C. Cordes, Nucleoporins as Components of the Nuclear Pore Complex Core Structure and Tpr as the Architectural Element of the Nuclear Basket, Mol. Biol. Cell. 15 (2004) 4261-4277. doi:10.1091/mbc.E04.

[13] B. Vollmer, W. Antonin, The diverse roles of the Nup93 / Nic96 complex proteins - structural scaffolds of the nuclear pore complex with additional cellular functions, Biol. Chem. 395 (2014) 515528. doi:10.1515/hsz-2013-0285.

[14] A. Von Appen, J. Kosinski, L. Sparks, A. Ori, A.L. Diguilio, B. Vollmer, M. Mackmull, N. Banterle, L. Parca, P. Kastritis, K. Buczak, S. Mosalaganti, W. Hagen, A. Andres-pons, E.A. Lemke, P. Bork, W. Antonin, J.S. Glavy, K.H. Bui, M. Beck, In situ structural analysis of the human nuclear pore complex, Nature. 526 (2015) 140-143. doi:10.1038/nature15381.

[15] J. Kosinski, S. Mosalaganti, A. Von Appen, R. Teimer, A.L. Diguilio, W. Wan, K.H. Bui, W.J.H. Hagen, J. a G. Briggs, J.S. Glavy, E. Hurt, M. Beck, Molecular architecture of the inner ring scaffold of the human nuclear pore complex, Science. 352 (2016) 363-365.

[16] D.P. Denning, S.S. Patel, V. Uversky, A.L. Fink, M. Rexach, Disorder in the nuclear pore complex: the FG repeat regions of nucleoporins are natively unfolded., Proc. Natl. Acad. Sci. U. S. A. 100 (2003) 2450-2455. doi:10.1073/pnas.0437902100.

[17] M. Kampmann, G. Blobel, Three-dimensional structure and flexibility of a membrane-coating module of the nuclear pore complex, Nat. Struct. Mol. Biol. 16 (2009) 782-789. 
doi:10.1038/nsmb.1618.

[18] J. Fiserova, E. Kiseleva, M.W. Goldberg, Nuclear envelope and nuclear pore complex structure and organization in tobacco BY-2 cells., Plant J. 59 (2009) 243-255. doi:10.1111/j.1365313X.2009.03865.x.

[19] N. Neumann, D. Lundin, A.M. Poole, Comparative genomic evidence for a complete nuclear pore complex in the last eukaryotic common ancestor, PLoS One. 5 (2010). doi:10.1371/journal.pone.0013241.

[20] M.C. Field, L. Koreny, M.P. Rout, Enriching the Pore: Splendid Complexity from Humble Origins, Traffic. 15 (2014) 141-156. doi:10.1111/tra.12141.

[21] K.E. Knockenhauer, T.U. Schwartz, The Nuclear Pore Complex, Cell. 164 (2016) 1162-1171. doi:10.1016/j.cell.2016.01.034.

[22] F. Alber, S. Dokudovskaya, L.M. Veenhoff, W. Zhang, J. Kipper, D. Devos, A. Suprapto, O. KarniSchmidt, R. Williams, B.T. Chait, A. Sali, M.P. Rout, The molecular architecture of the nuclear pore complex, Nature. 450 (2007) 695-701. doi:10.1038/nature06405.

[23] S.S. Patel, B.J. Belmont, J.M. Sante, M.F. Rexach, Natively unfolded nucleoporins gate protein diffusion across the nuclear pore complex., Cell. 129 (2007) 83-96. doi:10.1016/j.cell.2007.01.044.

[24] S. Frey, D. Görlich, A Saturated FG-Repeat Hydrogel Can Reproduce the Permeability Properties of Nuclear Pore Complexes, Cell. 130 (2007) 512-23. doi:10.1016/j.cell.2007.06.024.

[25] R. Peters, Translocation through the nuclear pore: Kaps pave the way, BioEssays. 31 (2009) 466-477. doi:10.1002/bies.200800159.

[26] J. Yamada, J.L. Phillips, S. Patel, G. Goldfien, A. Calestagne-Morelli, H. Huang, R. Reza, J. Acheson, V. V Krishnan, S. Newsam, A. Gopinathan, E.Y. Lau, M.E. Colvin, V.N. Uversky, M.F. Rexach, A Bimodal Distribution of Two Distinct Categories of Intrinsically Disordered Structures with Separate Functions in FG Nucleoporins, Mol. Cell. Proteomics. 9 (2010) 2205-2224. doi:10.1074/mcp.M000035MCP201.

[27] J. Ma, A. Goryaynov, A. Sarma, W. Yang, Self-regulated viscous channel in the nuclear pore complex, Proc. Natl. Acad. Sci. U. S. A. 109 (2012) 7326-31. doi:10.1073/pnas.1201724109.

[28] A. Bestembayeva, A. Kramer, A. Labokha, D. Osmanović, I. Liashkovich, E. V Orlova, I.J.. Ford, G. Charras, A. Fassati, B.W. Hoogenboom, Nanoscale stiffness topography reveals structure and mechanics of the transport barrier in intact nuclear pore complexes, Nat. Nanotechnol. 10 (2014) 60-64. doi:10.1038/nnano.2014.262.

[29] B.L. Timney, B. Raveh, R. Mironska, J.M. Trivedi, S.J. Kim, D. Russel, S.R. Wente, A. Sali, M.P. Rout, Simple rules for passive diffusion through the nuclear pore complex, J. Cell Biol. 215 (2016) jcb.201601004. doi:10.1083/jcb.201601004.

[30] V.N. Uversky, A.K. Dunker, Understanding protein non-folding, Biochim. Biophys. Acta - Proteins Proteomics. 1804 (2010) 1231-1264. doi:10.1016/j.bbapap.2010.01.017.

[31] L.E. Hough, K. Dutta, S. Sparks, D.B. Temel, A. Kamal, M.P. Rout, D. Cowburn, The molecular mechanism of nuclear transport revealed by atomic-scale measurements, Elife. (2015) 1-23. doi:10.7554/eLife.10027.

[32] N.B. Eisele, A.A. Labokha, S. Frey, D. Görlich, R.P. Richter, Cohesiveness tunes assembly and morphology of FG nucleoporin domain meshworks - Implications for nuclear pore permeability, Biophys. J. 105 (2013) 1860-1870. doi:10.1016/j.bpj.2013.09.006.

[33] A.R. Lowe, J.H. Tang, J. Yassif, M. Graf, W.Y.C. Huang, J.T. Groves, K. Weis, J.T. Liphardt, Importinbeta modulates the permeability of the nuclear pore complex in a Ran-dependent manner, Elife. 2015 (2015) 1-24. doi:10.7554/eLife.04052.

[34] A. Loschberger, C. Franke, G. Krohne, S. van de Linde, M. Sauer, Correlative super-resolution fluorescence and electron microscopy of the nuclear pore complex with molecular resolution, J. Cell Sci. 127 (2014) 4351-4355. doi:10.1242/jcs.156620.

[35] S.R. Wente, M.P. Rout, The nuclear pore complex and nuclear transport, Cold Spring Harb. Perspect. Biol. (2010) 1-20. doi:10.1101/cshperspect.a000562.

[36] D. Görlich, U. Kutay, Transport between the cell nucleus and the cytoplasm., Annu. Rev. Cell Dev. Biol. 15 (1999) 607-60. doi:10.1146/annurev.cellbio.15.1.607. 
[37] R. Bayliss, T. Littlewood, M. Stewart, Structural Basis for the Interaction between FxFG Nucleoporin Repeats and Importin-beta in Nuclear Trafficking, Cell. 102 (2000) 99-108. doi:10.1016/S00928674(00)00014-3.

[38] T.A. Isgro, K. Schulten, Binding Dynamics of Isolated Nucleoporin Repeat Regions to Importin-beta, Structure. 13 (2005) 1869-1879. doi:10.1016/j.str.2005.09.007.

[39] R. Bayliss, S.W. Leung, R.P. Baker, B.B. Quimby, A.H. Corbett, M. Stewart, Structural basis for the interaction between NTF2 and nucleoporin FxFG repeats, EMBO J. 21 (2002) 2843-2853.

[40] S. Milles, D. Mercadante, V. Aramburu, M. Blackledge, E.A. Lemke, Plasticity of an Ultrafast Interaction between Nucleoporins and Nuclear Transport Receptors, Cell. 163 (2015) 734-745. doi:10.1016/j.cell.2015.09.047.

[41] A. Paradise, M.K. Levin, G. Korza, J.H. Carson, Significant Proportions of Nuclear Transport Proteins with Reduced Intracellular Mobilities Resolved by Fluorescence Correlation Spectroscopy, J. Mol. Biol. 365 (2007) 50-65. doi:10.1016/j.jmb.2006.09.089.

[42] R. Zahn, D. Osmanović, S. Ehret, C. Araya Callis, S. Frey, M. Stewart, C. You, D. Görlich, B.W. Hoogenboom, R.P. Richter, A physical model describing the interaction of nuclear transport receptors with FG nucleoporin domain assemblies, Elife. 5 (2016) 1-21. doi:10.7554/eLife.14119.

[43] T. Jovanovic-Talisman, J. Tetenbaum-Novatt, A.S. McKenney, A. Zilman, R. Peters, M.P. Rout, B.T. Chait, Artificial nanopores that mimic the transport selectivity of the nuclear pore complex, Nature. 457 (2009) 1023-7. doi:10.1038/nature07600.

[44] L.E. Kapinos, R.L. Schoch, R.S. Wagner, K.D. Schleicher, R.Y.H. Lim, Karyopherin-centric control of nuclear pores based on molecular occupancy and kinetic analysis of multivalent binding with FG nucleoporins, Biophys. J. 106 (2014) 1751-1762. doi:10.1016/j.bpj.2014.02.021.

[45] R.S. Wagner, L.E. Kapinos, N.J. Marshall, M. Stewart, R.Y.H. Lim, Promiscuous binding of karyopherin-beta1 modulates FG nucleoporin barrier function and expedites NTF2 transport kinetics, Biophys. J. 108 (2015) 918-927. doi:10.1016/j.bpj.2014.12.041.

[46] B. Fahrenkrog, U. Aebi, The nuclear pore complex: nucleocytoplasmic transport and beyond, Nat. Rev. Mol. Cell Biol. 4 (2003) 757-66. doi:10.1038/nrm1230.

[47] A. Hoelz, E.W. Debler, G. Blobel, The Structure of the Nuclear Pore Complex, Annu. Rev. Biochem. 80 (2011) 613-643. doi:10.1146/annurev-biochem-060109-151030.

[48] H. Oberleithner, E. Brinckmann, a Schwab, G. Krohne, Imaging nuclear pores of aldosteronesensitive kidney cells by atomic force microscopy., Proc. Natl. Acad. Sci. U. S. A. 91 (1994) 9784-8. doi:10.1073/pnas.91.21.9784.

[49] J.O. Bustamante, A. Liepins, R.A. Prendergast, J.A. Hanover, H. Oberleithner, Membrane biology patch clamp and atomic force microscopy demonstrate TATA-binding protein (TBP) interactions with the nuclear pore complex, J. Membr. Biol. 272 (1995) 263-272.

[50] S. Schneider, G. Folprecht, G. Krohne, H. Oberleithner, Immunolocalization of lamins and nuclear pore complex proteins by atomic force microscopy, Pflügers Arch. Eur. J. Physiol. 430 (1995) 795801.

[51] T. Danker, M. Mazzanti, R. Tonini, A. Rakowska, H. Oberleithner, Using atomic force microscopy to investigate patch-clamped nuclear membrane., Cell Biol. Int. 21 (1997) 747-757. doi:10.1006/cbir.1997.0219.

[52] A. Rakowska, T. Danker, S.W. Schneider, H. Oberleithner, ATP-induced shape change of nuclear pores visualized with the atomic force microscope, J. Membr. Biol. 163 (1998) 129-136. doi:10.1007/s002329900377.

[53] H. Wang, D.E. Clapham, Conformational changes of the in situ nuclear pore complex., Biophys. J. 77 (1999) 241-7. doi:10.1016/S0006-3495(99)76885-2.

[54] T. Danker, H. Oberleithner, Nuclear pore function viewed with atomic force microscopy, Pflügers Arch. Eur. J. Physiol. 439 (2000) 671-681. doi:10.1007/s004240050992.

[55] V. Shahin, T. Danker, K. Enss, R. Ossig, H. Oberleithner, Evidence for Ca2+ - and ATP-sensitive peripheral channels in nuclear pore complexes, FASEB J. 15 (2001) 1895-1901. doi:10.1096/fj.000838com.

[56] R.D. Jäggi, A. Franco-Obregón, P. Mühlhäusser, F. Thomas, U. Kutay, K. Ensslin, Modulation of 
Nuclear Pore Topology by Transport Modifiers., Biophys. J. 84 (2003) 665-70. doi:10.1016/S00063495(03)74886-3.

[57] R.D. Jäggi, A. Franco-Obregón, K. Ensslin, Quantitative topographical analysis of nuclear pore complex function using scanning force microscopy., Biophys. J. 85 (2003) 4093-8. doi:10.1016/S0006-3495(03)74821-8.

[58] V. Shahin, L. Albermann, H. Schillers, L. Kastrup, C. Schäfer, Y. Ludwig, C. Stock, H. Oberleithner, Steroids dilate nuclear pores imaged with atomic force microscopy, J. Cell. Physiol. 202 (2005) 591601. doi:10.1002/jcp.20152.

[59] A. Kramer, Y. Ludwig, V. Shahin, H. Oberleithner, A pathway separate from the central channel through the nuclear pore complex for inorganic ions and small macromolecules, J. Biol. Chem. 282 (2007) 31437-31443. doi:10.1074/jbc.M703720200.

[60] A. Kramer, I. Liashkovich, Y. Ludwig, V. Shahin, Atomic force microscopy visualises a hydrophobic meshwork in the central channel of the nuclear pore, Pflügers Arch. Eur. J. Physiol. 456 (2008) 155162. doi:10.1007/s00424-007-0396-y.

[61] A. Kramer, I. Liashkovich, H. Oberleithner, S. Ludwig, I. Mazur, V. Shahin, Apoptosis leads to a degradation of vital components of active nuclear transport and a dissociation of the nuclear lamina., Proc. Natl. Acad. Sci. U. S. A. 105 (2008) 11236-41. doi:10.1073/pnas.0801967105.

[62] Y. Sakiyama, A. Mazur, L.E. Kapinos, R.Y.H. Lim, Spatiotemporal dynamics of the nuclear pore complex transport barrier resolved by high-speed atomic force microscopy, Nat. Nanotechnol. (2016) 1-6. doi:10.1038/nnano.2016.62.

[63] K. Ribbeck, D. Görlich, Kinetic analysis of translocation through nuclear pore complexes., EMBO J. 20 (2001) 1320-30. doi:10.1093/emboj/20.6.1320.

[64] S. Frey, R.P. Richter, D. Görlich, FG-rich repeats of nuclear pore proteins form a three-dimensional meshwork with hydrogel-like properties, Science. 314 (2006) 815-7. doi:10.1126/science.1132516.

[65] S. Frey, D. Görlich, FG/FxFG as well as GLFG repeats form a selective permeability barrier with selfhealing properties, Embo J. 28 (2009) 2554-2567. doi:DOI 10.1038/emboj.2009.199.

[66] S. Milles, E.A. Lemke, Single molecule study of the intrinsically disordered FG-repeat nucleoporin 153, Biophys. J. 101 (2011) 1710-1719. doi:10.1016/j.bpj.2011.08.025.

[67] B.B. Hülsmann, A.A. Labokha, D. Görlich, The permeability of reconstituted nuclear pores provides direct evidence for the selective phase model, Cell. 150 (2012) 738-751. doi:10.1016/j.cell.2012.07.019.

[68] A.A. Labokha, S. Gradmann, S. Frey, B.B. Hülsmann, H. Urlaub, M. Baldus, D. Görlich, Systematic analysis of barrier-forming FG hydrogels from Xenopus nuclear pore complexes, EMBO J. 32 (2013) 204-18. doi:10.1038/emboj.2012.302.

[69] M.P. Rout, J.D. Aitchison, A. Suprapto, K. Hjertaas, Y. Zhao, B.T. Chait, The Yeast Nuclear Pore Complex, J. Cell Biol. 148 (2000) 635-652. doi:10.1083/jcb.148.4.635.

[70] M.P. Rout, J.D. Aitchison, M.O. Magnasco, B.T. Chait, Virtual gating and nuclear transport: The hole picture, Trends Cell Biol. 13 (2003) 622-628. doi:10.1016/j.tcb.2003.10.007.

[71] R.Y.H. Lim, N.-P.P. Huang, J. Köser, J. Deng, K.H.A. Lau, K. Schwarz-Herion, B. Fahrenkrog, U. Aebi, Flexible phenylalanine-glycine nucleoporins as entropic barriers to nucleocytoplasmic transport, Proc. Natl. Acad. Sci. 103 (2006) 9512-9517. doi:10.1073/pnas.0603521103.

[72] R. Peters, Translocation Through the Nuclear Pore Complex: Selectivity and Speed by Reduction-ofDimensionality, Traffic. 6 (2005) 421-427. doi:10.1111/j.1600-0854.2005.00287.x.

[73] R.Y.H. Lim, B. Fahrenkrog, J. Köser, K. Schwarz-Herion, J. Deng, U. Aebi, Nanomechanical Basis of Selective Gating by the Nuclear Pore Complex, Science. 318 (2007) 640-3. doi:10.1126/science.1145980.

[74] R.L. Schoch, L.E. Kapinos, R.Y.H. Lim, Nuclear transport receptor binding avidity triggers a selfhealing collapse transition in FG-nucleoporin molecular brushes., Proc. Natl. Acad. Sci. U. S. A. 109 (2012) 16911-6. doi:10.1073/pnas.1208440109.

[75] A. Vovk, C. Gu, M.G. Opferman, L.E. Kapinos, R.Y.H. Lim, R.D. Coalson, D. Jasnow, A. Zilman, Simple biophysics underpins collective conformations of the intrinsically disordered proteins of the Nuclear Pore Complex, Elife. 5 (2016) 1-29. doi:10.7554/eLife.10785. 
[76] N.B. Eisele, S. Frey, J. Piehler, D. Görlich, R.P. Richter, Ultrathin nucleoporin phenylalanine-glycine repeat films and their interaction with nuclear transport receptors, EMBO Rep. 11 (2010) 366-72. doi:10.1038/embor.2010.34.

[77] B. Fahrenkrog, E.C. Hurt, U. Aebi, N. Panté, Molecular architecture of the yeast nuclear pore complex: Localization of Nsp1p subcomplexes, J. Cell Biol. 143 (1998) 577-588. doi:10.1083/jcb.143.3.577.

[78] B. Fahrenkrog, J.P. Aris, E.C. Hurt, N. Panté, U. Aebi, Comparative Spatial Localization of Protein-ATagged and Authentic Yeast Nuclear Pore Complex Proteins by Immunogold Electron Microscopy, J. Struct. Biol. 129 (2000) 295-305. doi:10.1006/jsbi.2000.4223.

[79] I. Ben-Efraim, L. Gerace, Gradient of Increasing Affinity of Importin beta for Nucleoporins along the Pathway of Nuclear Import, J. Cell Biol. 152 (2001) 411-417.

[80] J. Bednenko, G. Cingolani, L. Gerace, Importin beta contains a $\mathrm{COOH}$-terminal nucleoporin binding region important for nuclear transport, J. Cell Biol. 162 (2003) 391-401. doi:10.1083/jcb.200303085.

[81] D. Osmanović, J. Bailey, A.H. Harker, A. Fassati, B.W. Hoogenboom, I.J. Ford, Bistable collective behavior of polymers tethered in a nanopore, Phys. Rev. 61917 (2012) 1-8. doi:10.1103/PhysRevE.85.061917.

[82] D. Osmanović, I.J. Ford, B.W. Hoogenboom, Model Inspired by Nuclear Pore Complex Suggests Possible Roles for Nuclear Transport Receptors in Determining Its Structure, Biophys. J. 105 (2013) 2781-2789. doi:10.1016/j.bpj.2013.11.013.

[83] O. Peleg, M. Tagliazucchi, M. Kröger, Y. Rabin, I. Szleifer, Morphology control of hairy nanopores, ACS Nano. 5 (2011) 4737-4747. doi:10.1021/nn200702u.

[84] N. Panté, M. Kann, Nuclear Pore Complex Is Able to Transport Macromolecules with Diameters of 39 nm, Mol. Biol. Cell. 13 (2002) 425-434. doi:10.1091/mbc01.

[85] D. Osmanović, A. Fassati, I.J. Ford, B.W. Hoogenboom, Physical modelling of the nuclear pore complex, Soft Matter. 9 (2013) 10442-10451. doi:10.1039/c3sm50722j.

[86] H.B. Schmidt, D. Görlich, Nup98 FG domains from diverse species spontaneously phase-separate into particles with nuclear pore-like permselectivity., Elife. 4 (2015) 1-30. doi:10.7554/eLife.04251.

[87] S.R. Solmaz, R. Chauhan, G. Blobel, I. Melčák, Molecular architecture of the transport channel of the nuclear pore complex, Cell. 147 (2011) 590-602. doi:10.1016/j.cell.2011.09.034. 